\title{
Complexity and approximation for scheduling problem for a torpedo
}

\author{
G. Simonin ${ }^{1}$, R. Giroudeau ${ }^{1}$, and J.C. König ${ }^{1}$ \\ ${ }^{1}$ LIRMM, 161 rue Ada, 34392 Montpellier Cedex 5, France, UMR 5506
}

\begin{abstract}
This paper considers a special case of the coupled-tasks scheduling problem on one processor. The general problems were analyzed in depth by Orman and Potts [1]. In this paper, we consider that all processing times are equal to 1, the gap has exact length $L$, we have precedence constraints, compatibility constraints are introduced and the criterion is to minimize the scheduling length. We use this problem to study the problem of data acquisition and data treatment of a torpedo under the water. We show that this problem is $\mathcal{N} \mathcal{P}$-complete and we propose an $\rho$-approximation algorithm where $\rho \leq \frac{(L+6)}{6}$.

Keywords: scheduling, coupled-tasks, compatibility constraints, complexity, approximation
\end{abstract}

\section{Introduction}

\subsection{Presentation}

In this paper, we present the problem of data acquisition according to compatibility constraints in a submarine torpedo, denoted TORPEDO problem. The torpedo is used in order to make cartography, topology studies, temperature measures and many other tasks in the water. The aim of this torpedo is to collect and process a set of data as soon as possible on a mono processor. In this way, it possess few sensors, a mono processor and two types of tasks which must be schedule: Acquisition tasks and treatment tasks.

First, the acquisition tasks $\mathcal{A}=\left\{A_{1}, \ldots, A_{n}\right\}$ can be assigned to coupled-tasks introduced by Shapiro [2], indeed the torpedo sensors emit a wave which propagates in the water in order to collect the data. Each acquisition tasks $A_{i}$ have two sub-tasks, the first $a_{i}$ sends an echo, the second $b_{i}$ receives it. For a better reading, we will denote the processing time of each sub-task $a_{i}$ and $b_{i}$. Between the sub-tasks, there is an incompressible idle time $L_{i}$ which represents the spread of the echo in the water.

Second, treatment tasks $\mathcal{T}=\left\{T_{1}, \ldots, T_{n}\right\}$ are obtained from acquisition tasks, indeed after the return of the echo, various calculations will be executed from gathered informations. These tasks are preemptive and have precedence constraints with the acquisition tasks. In this paper, we will study the problem where every acquisition task have a precedence relation with only one treatment task.

At last, there exist compatibility constraints between acquisition tasks, due to the fact that some acquisition tasks cannot be processed in same the time that another tasks. In order to represent this constraint, a compatibility graph $G_{c}=\left(\mathcal{A}, E_{c}\right)$ is introduced, where $\mathcal{A}$ is the set of coupledtasks and $E_{c}$ represents the edges which link two coupledtasks which can be executed simultaneously. In other words, at least one sub-task of a task $A_{i}$ may be executed during the idle time of another task $A_{j}$ (see example in Figure 1).
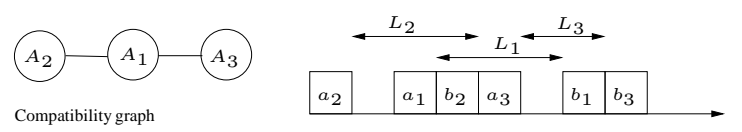

Fig. 1: Example of compatibility constraints with $L_{1}=L_{2}=3$ and $L_{3}=2$

The aim of the TORPEDO problem is to produce a shortest schedule (i.e. to minimize the moment after the execution of the last task in the schedule denoted $C_{\max }$ ) in which compatibility contraints between acquisition tasks and precedence constraints are respected. In scheduling theory, a problem is categorized by its machine environment, job characteristic and objective function. So using the notation scheme $\alpha|\beta| \gamma$ proposed by [3], the TORPEDO problem will be defined by $1 \mid$ prec, $\left(a_{i}, L_{i}, b_{i}\right) \cup$ $\left(T_{i}, p m t n\right), G_{c} \mid C_{\max }{ }^{1}$.

Our work consists in measuring the impact of the compatibility graph on the complexity and approximation of scheduling problems with coupled-tasks on a mono processor. This paper is focusing on the limit between polynomial problems and $\mathcal{N} \mathcal{P}$-complete problems, when the compatibility constraint is introduced.

\subsection{Related work}

The complexity of the scheduling problem, with coupledtasks and a complete compatibility graph ${ }^{2}$, has been investigated by Blazewicz and al. [4], Orman and Potts [1], Ahr and al. [5]. Nevertheless, in the article we study a different problem in which coupled-tasks (or acquisition tasks) must respect a compatibility graph. Morever, in our model, we consider a set of treatment tasks whose have a precedence constraint with the set of acquisition tasks, whereas in existing works the authors ([4],[1],[5]) focus their studies on precedence constraints between the acquisition tasks. By comparing the results of Orman and Potts [1] and those obtained by relaxing the constraint of compatibility, we can measure the impact of compatibility constraint on this kind of problem.

\footnotetext{
${ }^{1}$ prec (resp. pmtn) represents the precedence constraints between $\mathcal{A}$ et $\mathcal{T}$ (resp. the preemtivity of the treatment tasks)

${ }^{2}$ Notice, the lack of compatibility graph is equivalent to a fully connected graph. In this way, all tasks may be compatible each other.
} 


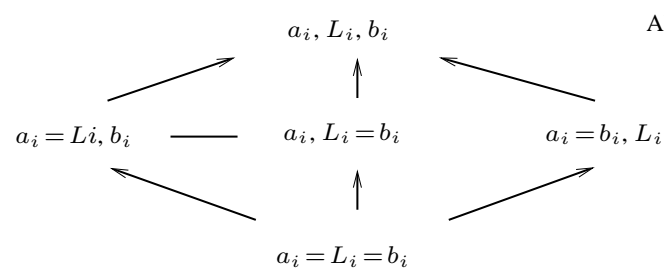

ALL $\mathcal{N} \mathcal{P}$-COMPLETES PROBLEMS
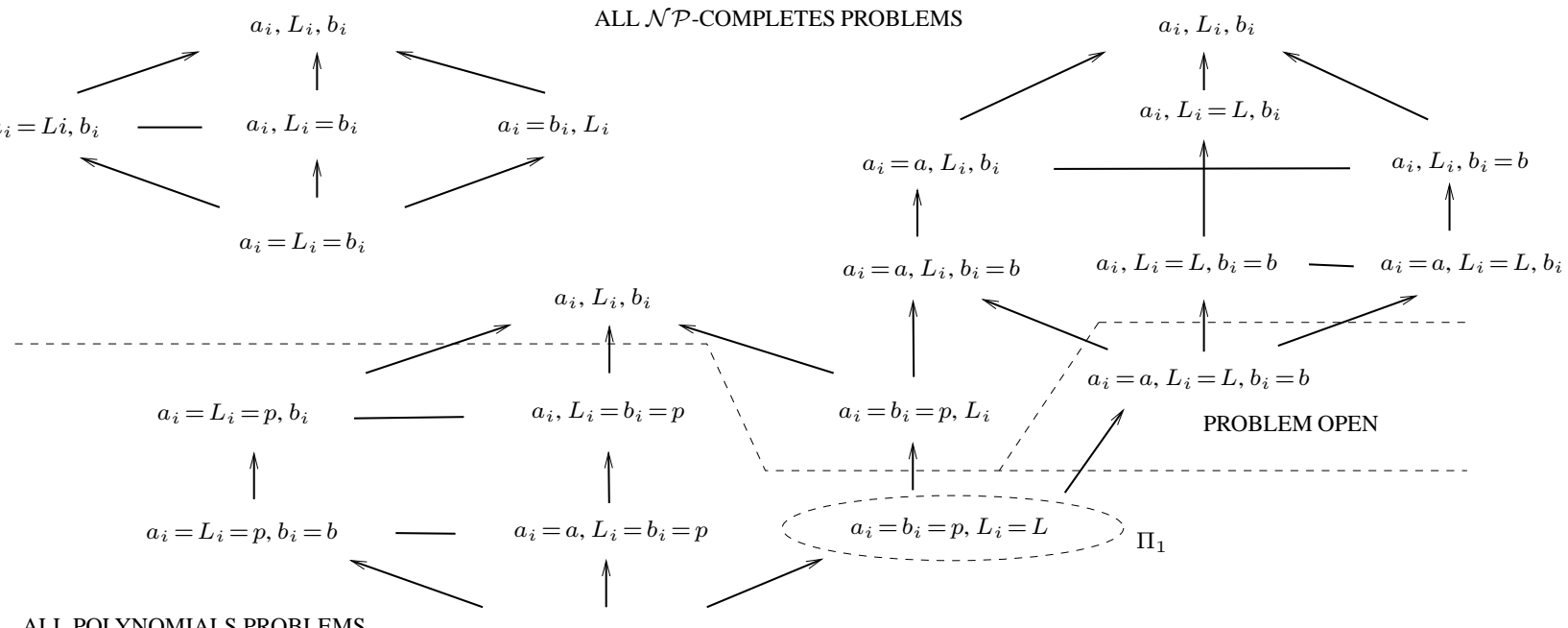

ALL POLYNOMIALS PROBLEMS

$a_{i}=a, L_{i}=L, b_{i}=b$

Fig. 2: Global visualisation of the complexity of scheduling problems with coupled-tasks given by Orman and Potts [1]. The graphs are described like this: $a_{i}, L_{i}, b_{i}, G_{c}$ represents the type of problem studied, where $a_{i}, b_{i}$ and $L_{i}$ can take any value or be all equal to a constant. Finally, there is an arc from a specific problem to a more general problem, and an edge between two symmetrical problems.

We derive two main results: First, starting from the complexity results of Orman and Potts resumed in Figure 2, we show the complexity of a special problem, denoted $\Pi_{1}$ which becomes $\mathcal{N} \mathcal{P}$-complete when the compatibility constraint is relaxed. The $\mathcal{N} \mathcal{P}$-completeness of $\Pi_{1}$ imply the $\mathcal{N P}$-completeness of all the problems which are more general (see Figure 2). Second, we develop a polynomial-time approximation algorithm based on a maximum matching on the compatibility graph for $\Pi_{1}$.

This article is organized as follows: In the next section, we will prove the $\mathcal{N} \mathcal{P}$-completeness of $\Pi_{1}=1 \mid$ prec, $\left(a_{i}=\right.$ $\left.b_{i}=p, L_{i}=L, G_{c}\right) \cup\left(T_{i}\right.$, pmtn $) \mid C_{\max }$ where the two subtasks $a_{i}$ and $b_{i}$ are equal to a constant $p$ and the inactivity time $L_{i}$ is equal to a constant $L$ (the reduction is based from the $\mathcal{N} \mathcal{P}$-complete Clique, Garey and Johnson [6] GT19), and so $t_{b_{i}}=t_{a_{i}}+a_{i}+L_{i}=t_{a_{i}}+p+L$ where $t_{a_{i}}$ (resp. $t_{b_{i}}$ ) is the starting time of the sub-task $a_{i}$ (resp. $t_{b_{i}}$ ). In the last section, we develop a polynomial-time approximation algorithm for $\Pi_{1}$ with performance guarantee less than $\frac{L+6}{6}$.

\section{Complexity result}

In this section, notice that in the special case where $L=1$, the problem is polynomial. It is sufficient to find a maximum matching in the compatibility graph. We focus the case $L \neq 1$ and $L$ is a data of the problem. The case where $L=2$, is studied in another paper [7]. In order to prove the $\mathcal{N} \mathcal{P}$-completeness of $\Pi_{1}$, we will prove the $\mathcal{N} \mathcal{P}$-completeness of the specific case $\Pi_{2}=1 \mid$ prec, $\left(a_{i}=\right.$ $\left.b_{i}=1, L_{i}=L\right) \cup\left(T_{i}\right.$, pmtn $), G_{c} \mid C_{\max }$.

Theorem 2.1 Let $n$ be the acquisition tasks number, the problem, to decide if an instance of the problem $\Pi_{2}$ has a scheduling length $C_{\max }=2 n+\sum_{T_{i} \in \mathcal{T}} T_{i}$, is $\mathcal{N} \mathcal{P}$. complete.

\section{Proof}

Our approach is similar to the proof of Lenstra and Rinnoy Kan [3] for the problem $P \mid$ prec; $p_{j}=1 \mid C_{\max }$. This demonstration is based on the Clique decision problem (see Garey and Johnson GT19 [6]):

INSTANCE: A graph $G=(V, E)$ where $|V|=n$, and an integer $K$.

QUESTION: Can we find a clique of size $K$ in $G$ ?

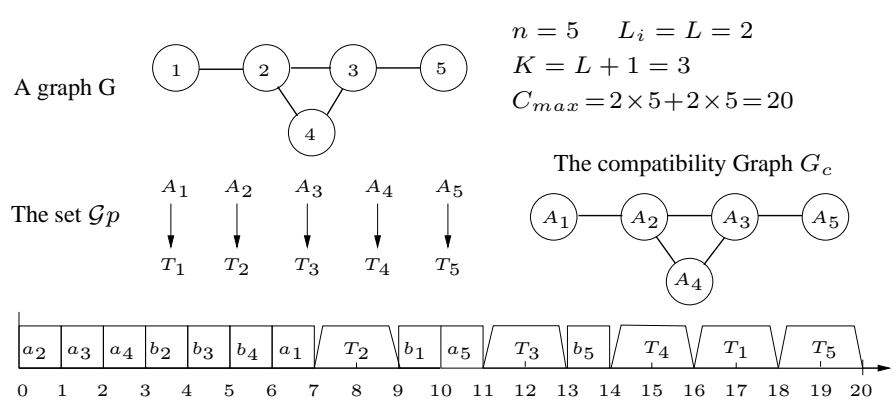

Fig. 3: Illustration of polynomial-time transformation Clique $\propto \Pi_{2}$

Our proof is based on the polynomial-time transformation Clique $\propto \Pi_{2}$. It is easy to see that the problem $\Pi_{2}$ is in $\mathcal{N P}$.

Let $I^{*}$ an instance of Clique, we will construct an instance $I$ of $\Pi_{2}$ with $C_{\max }=2 n+\sum_{T_{i} \in \mathcal{T}} T_{i}$ in the following way:

Let $G=(V, E)$ a graph in the instance $I$, with $|V|=n$ :

- $\forall v \in V$, an acquisition task $A_{v}$ is introduced, composed of two sub-tasks $a_{v}$ and $b_{v}$ with processing time $a_{v}=b_{v}=1$ and with a latency time $L_{v}$, between these two sub-tasks, of length $L=(K-1)$, called slot.

- For each edge $e=(v, w) \in E$, there is a compatibility relation between the two acquisition tasks $A_{v}$ and $A_{w}$. 
- For each task $A_{v}$, we introduce a treatment task $T_{v}$ which is its successor.

- Each $T_{v}$ has a processing time noted $T_{v}=L$. Thus, the treatment tasks will replace all the inactivity slot of all the $A_{v}$ after the clique.

- We suppose that there is a clique of length $K=(L+1)$ in the graph $G$. Let us show that there is a scheduling in $C_{\max }=\left(2 n+\sum_{T_{i} \in \mathcal{T}} T_{i}\right)$ units of time. For that, consider the following scheduling:

- From time $t=0$ to $t=L$, we schedule the $K=$ $(L+1)$ tasks which represent the vertices of the clique of size $K$.

- From time $t=(2 L+2)$, we schedule the $(n-K)$ remaining tasks $A_{v}$.

- In each slot from these $(n-K)$ tasks $A_{v}$, we schedule the tasks $T_{v}$. Since each $T_{v}$ has as a value $T_{v}=L$, by scheduling $(n-K)$ tasks $T_{v}$, we will fill each slot of length $L$ of the $(n-K)$ tasks $A_{v}$.

- Remeaning treatment tasks are scheduled at the end of the schedule.

With this allocation, we fill all the slots and we give a valid scheduling in $\left(2 n+\sum_{T_{i} \in \mathcal{T}} T_{i}\right)$ units of time.

- Reciprocally, let us suppose that there is a scheduling in $\left(2 n+\sum_{T_{i} \in \mathcal{T}} T_{i}\right)$ units of time without inactivity time for $\Pi_{2}$, then let us show that the graph $G$ contains a clique of size $K=(L+1)$.

From these suppositions, we make essential comments:

- With the precedence constraints between the tasks $A_{v}$ and $T_{v}$, it is easy to see that we can schedule only tasks $A_{v}$ at $t=0, \forall v \in V$. Thus, the first treatment task could be scheduled only starting from $t=(L+2)$.

- Let $a_{p_{1}}$ be the first sub-task of acquisition scheduled at $t=0$, with a slot of length $L$. We need a clique of size $(L+1)$ to obtain a scheduling without inactivity slot.

Thus we have $(L+1)$ acquisition tasks which are compatibles. And in the compatibility graph $G_{c}$, we will have an edge between each couple of these tasks $A_{v}$. Consequently, the tasks $A_{p_{1}}, A_{p_{2}}, \ldots, A_{p_{L}}$, associated to the vertices of the graph $\mathrm{G}$, form a clique of size $K=(L+1)$.

This concludes our proof of Theorem 2.1.

From this result we can conclude of the NP-completeness of $\Pi_{1}$. With the global visualization of Figure 2 , we see that the $\mathcal{N} \mathcal{P}$-completeness of $\Pi_{1}$ imply the $\mathcal{N} \mathcal{P}$-completeness of all the more general problems. And thus, the open problem in the study of Orman and Potts [1] become $\mathcal{N} \mathcal{P}$-complete with the relaxation of the compatibility constraint.

\section{Approximation algorithm}

In this section, we will present and study a polynomialtime approximation algorithm for $\Pi_{2}$ based on maximum matching.

Remark 3.1 Notice that, in the case where processing time of treatment tasks is greater than one $\left(T_{i}>1, \forall i\right)$, then the sum of idle time in a schedule cannot be higher as if the processing time of treatment tasks is one $\left(T_{i}=1\right)$.

In the following, treatment tasks will have processing time equal to $T_{i}=1$. We will present an approximation algorithm of the problem $\Pi_{2}$.

\subsection{Lower bounds}

We will give two lower bounds. For the first, optimal scheduling is taken where we do not have any time of inactivity. Moreover we know that the number of treatment tasks is equal to the number of acquisition tasks and that in worst case all the treatment tasks have an processing time $T_{i}=1, \forall i$. Thus, we have:

$$
C_{\text {max }}^{\text {opt }} \geq T_{\text {seq }}=2 n+\sum_{T_{i} \in \mathcal{T}} T_{i} \geq 2 n+n=3 n
$$

For the second bound, the maximum matching is taken of the compatibility graph $G_{c}$, its cardinality is $m$, and thus we have $(n-2 m)$ independent vertices. In worst case, optimal scheduling is greater than independent vertices scheduling with the last treatment task. And so we have:

$$
C_{\text {max }}^{\text {opt }} \geq(n-2 m)(L+2)+1
$$

For our study, our lower bound will be

$$
C_{\max }^{o p t} \geq \max \{3 n,(n-2 m)(L+2)+1\}
$$

\subsection{Upper bound}

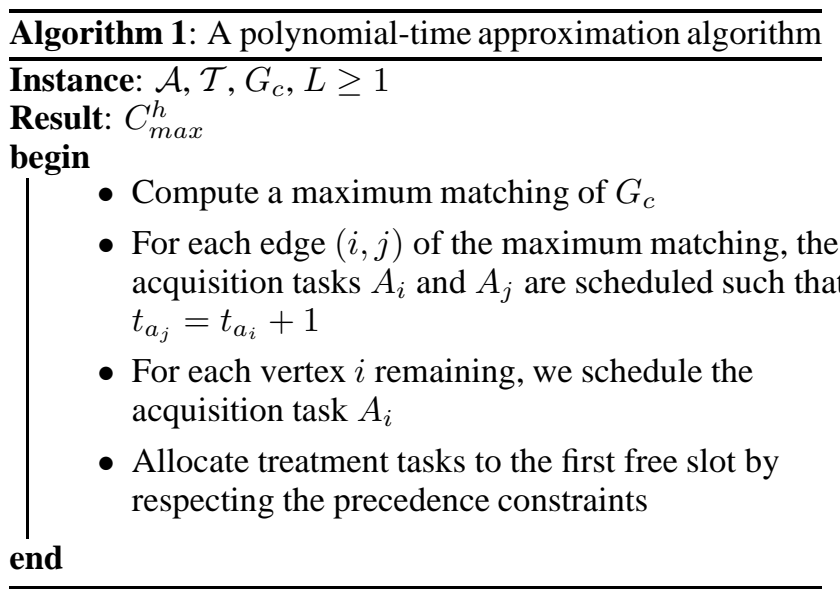

We will give some essential remarks on the structure of the scheduling given by our approximation algorithm. Let us suppose that we have a scheduling given by the approximation algorithm with a maximum matching of size $m$.

- In the first coupled-task matched, there is an incompressible latency length of size $(L-1)$. 


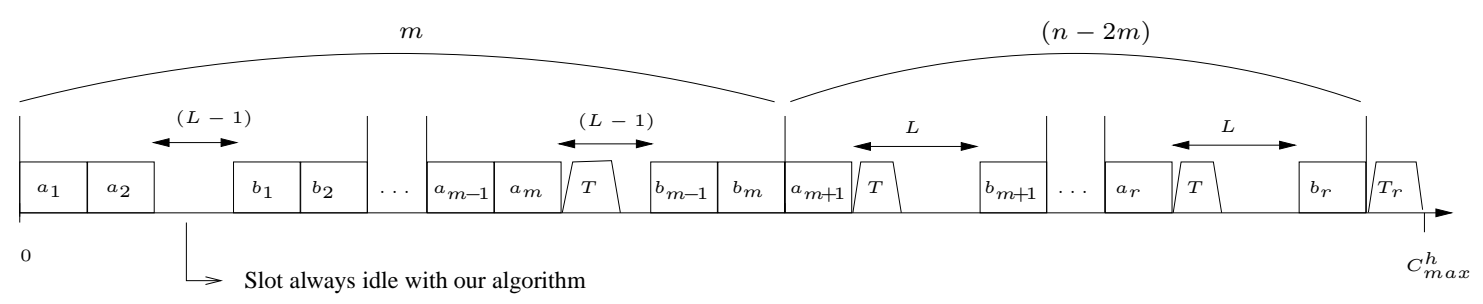

Fig. 4: Illustration of the approximation algorithm

- We have $n$ acquisition tasks, the scheduling length of these tasks is $2 n$.

- For two tasks matched, the incompressible latency length is $(L-1)$.

- For each remaining vertex, incompressible latency length is $L$.

- Considering the last acquisition task $A_{r}$. After its execution, we may process one treatment task denoted by $T_{r}$ (this case occurs when all the treatment tasks, except $T_{r}$, are scheduled before the completion time of $A_{r}$ ), or some treatment tasks (this case occurs when there is no idle time before the completion time of $A_{r}$ ). See figure (4), for an illustration of the case where the task $T_{r}$ is the only treatment task executed after the completion time of $A_{r}$.

So, the number of treatment task executed after $A_{r}$ is:

$$
\begin{array}{r}
\max \{n-(m-1)(L-1)-(n-2 m) L, 1\} \\
\quad=\max \{n-n L+m(L+1)+L-1,1\}
\end{array}
$$

Finally, our upper bound will be:

$$
\begin{aligned}
& C_{\max }^{h} \leq T_{\text {execution }}+T_{\text {incompressible latency length }} \\
& \leq[2 n+\max \{n-n L+m(L+1)+L-1,1\}] \\
& +[(L-1) m+L(n-2 m)] \\
& \leq[2 n+\max \{n-n L+m(L+1)+L-1,1\}] \\
& +[L(n-m)-m]
\end{aligned}
$$

\begin{tabular}{|c|c|c|}
\hline & $\alpha=1$ & $L=2$ \\
\hline$C_{\max }^{h}$ & $3 n$ & $\begin{array}{l}\text { if } m \geq \frac{n}{3} \text {, then } C_{\max }^{h} \leq 3 n+1 \\
\text { if } \frac{n+1}{8} \leq m<\frac{n}{3} \text {, then } C_{\max }^{h} \leq 4 n-3 m+1 \\
\text { if } m<\frac{n+1}{8} \text {, then } C_{\max }^{h} \leq 4 n-3 m+1\end{array}$ \\
\hline$C_{\max }^{o p t}$ & $3 n$ & $\begin{array}{l}\text { if } m \geq \frac{n}{3}, \text { then } C_{\max }^{o p t} \geq 3 n \\
\text { if } \frac{n+1}{8} \leq m<\frac{n}{3}, \text { then } C_{\max }^{o p t} \geq 3 n \\
\text { if } m<\frac{n+1}{8} \text {, then } C_{\max }^{o p t} \geq 4 n-8 m+1\end{array}$ \\
\hline$\rho$ & 1 & $\begin{array}{l}\text { if } m \geq \frac{n}{3} \text {, then } \rho \leq 1+\frac{1}{3 n} \\
\text { if } \frac{n+1}{8} \leq m<\frac{n}{3}, \text { then } \rho \leq \frac{4}{3}+\frac{1}{3 n} \\
\text { if } m<\frac{n+1}{8} \text {, then } \rho \leq \frac{11}{6}\end{array}$ \\
\hline
\end{tabular}

\subsection{Relative performance}

\begin{tabular}{|c|c|}
\hline & $L=3$ \\
\hline$C_{\max }^{h}$ & $\begin{array}{c}\text { if } m=\frac{n}{2} \text {, then } C_{\max }^{h} \leq 3 n+2 \\
\text { if } \frac{2 n+1}{10} \leq m<\frac{n}{2} \text {, then } C_{\max }^{h} \leq 5 n-4 m+1 \\
\text { if } m<\frac{2 n+1}{10} \text {, then } C_{\max }^{h} \leq 5 n-4 m+1\end{array}$ \\
\hline$C_{\max }^{o p t}$ & $\begin{array}{c}\text { if } m=\frac{n}{2}, \text { then } C_{\max }^{o p t} \geq 3 n \\
\text { if } \frac{2 n+1}{10} \leq m<\frac{n}{2}, \text { then } C_{\max }^{o p t} \geq 3 n \\
\text { if } m<\frac{2 n+1}{10} \text {, then } C_{\max }^{o p t} \geq 4 n-8 m+1\end{array}$ \\
\hline$\rho$ & $\begin{array}{c}\text { if } m=\frac{n}{2} \text {, then } \rho \leq \mathbf{1}+\frac{\mathbf{2}}{3 n} \\
\text { if } \frac{2 n+1}{10} \leq m<\frac{n}{2} \text {, then } \rho \leq \frac{5}{3}+\frac{1}{3 n} \\
\text { if } m<\frac{2 n+1}{10} \text {, then } \rho \leq \frac{21}{17}\end{array}$ \\
\hline
\end{tabular}

In the first step, the Tables 1 and 2 give a summarize of the ratio of relative performance $\rho \leq \frac{C_{m a x}^{h}}{C_{\max }^{p+t}}$ for $L \in\{1,2,3\}$.

Tab. 1: Relative performance for $L \in\{1,2,3\}$

Now we focuses on study for $L \geq 4$. Since that $m \leq \frac{n}{2}$, it easy to see that $\max \{n-n L+m(L+1)+L-1,1\} \stackrel{2}{=} 1$.
Tab. 2: Relative performance for $L \in\{1,2,3\}$

And so $C_{\max }^{h} \leq 2 n+L(n-m)-m+1$.

Moreover, since that $C_{\max }^{o p t} \geq \max \{3 n, n L+2 n-$ $2 m L-4 m+1\}$, the following cases must be considered:

- For $m \in\left[0, \frac{n(L-1)+1}{2(L+2)}\left[\right.\right.$, it is easy to see that $C_{\text {max }}^{\text {opt }} \geq$ $3 n$.

- For $m \in\left[\frac{n(L-1)+1}{2(L+2)}, \frac{n}{2}\right]$, we will have $C_{\max }^{o p t} \geq n L+$ $2 n-2 m L-4 m+1$.

According to the values of $m$, we give the upper bound for the length of the scheduling proposed by the heuristic $h$, and the lower bound for an optimal scheduling (see illustration figure 5).

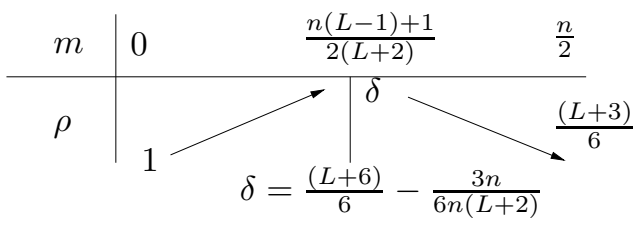

Fig. 5: Behavior of the relative performance $\rho$ as a fonction of $m$

Notice that for $m=0, \rho=1$ (it is clear, because the compatibility graph is an independent set), moreover for $m=\frac{n}{2}, \rho=\frac{(L+3)}{6}$. 


\section{Conclusion}

In this paper, we presented a scheduling problem on mono processor with graph constraints and coupled-tasks. On the negative side, we showed that the problem $\Pi_{2}$ is $\mathcal{N} \mathcal{P}$ complete, our proof is based on the polynomial-time transformation Clique to $\Pi_{2}$, and imply the $\mathcal{N} \mathcal{P}$-completeness of all the more general problems (specially for the open problem in Figure 2 which become $\mathcal{N} \mathcal{P}$-complete with the relaxation of the compatibility constraint).

On the positive side, we gave an approximation algorithm for $\Pi_{2}$ with relative performance bounded by $\rho \leq \frac{L+6}{6}$ in the worst case, where $L$ is the inactivity time of acquisition tasks. The relative performance value $\rho$ associated to the algorithm depends on the parameter $L$, which is one of the problem data. This remark brings a fundamental question: "Is that our problem admits an approximation algorithm with a performance guarantee equal to a constant value?". The problem would be then in the class $A P X$, but if we can show that it will never exist an approximation algorithm with a performance guarantee equal to a constant value, then our problem would be in the classnon - APX.

\section{REFERENCES}

[1] A.J. Orman and C.N. Potts. On the complexity of coupled-task scheduling. Discrete Applied Mathematics, 72:141-154, 1997.

[2] R.D. Shapiro. Scheduling coupled tasks. Naval Research Logistics Quarterly, 27:477-481, 1980.

[3] R.L. Graham, E.L. Lawler, J.K. Lenstra, and A.H.G. Rinnooy Kan. Optimization and approximation in deterministic sequencing and scheduling: a survey. Annals of Discrete Mathematics, 5:287-326, 1979.

[4] J. Blazewicz, K.H. Ecker, T. Kis, and M. Tanas. A note on the complexity of scheduling coupled-tasks on a single processor. Journal of the Brazilian Computer Society, 7(3):23-26, 2001.

[5] D. Ahr, J. Békési, G. Galambos, M. Oswald, and G. Reinelt. An exact algorithm for scheduling identical coupled-tasks. Mathematical Methods of Operations Research, 59:193-203(11), June 2004.

[6] M.R. Garey and D.S. Johnson. Computers and Intractability; $A$ Guide to the Theory of NPCompleteness. W. H. Freeman \& Co., New York, NY, USA, 1990.

[7] G. Simonin, R.Giroudeau, and J.-C. König. Extended matching problem for a coupled-tasks scheduling problem. Technical rapport, LIRMM, January 2009. 